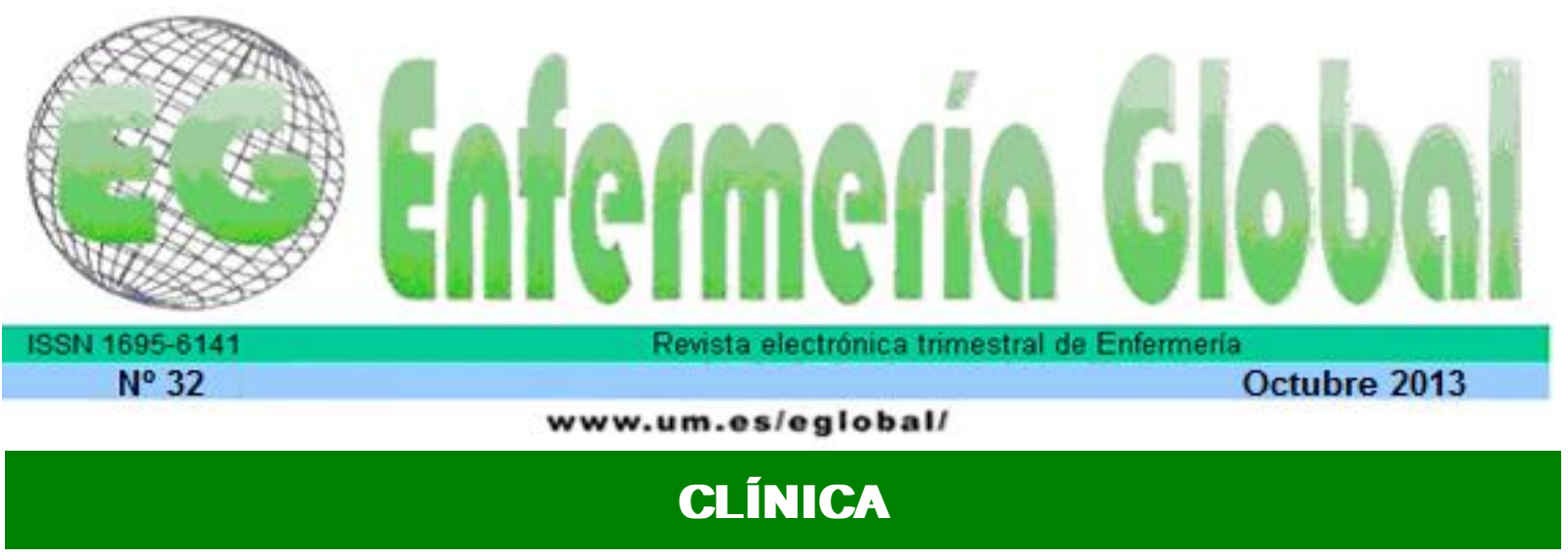

\title{
Uso del ácido Zoledrónico en el tratamiento del Mieloma Múltiple
}

Use of zoledronic acid in the treatment of Múltiple Myeloma

\section{*Hernández Cano, Rosa $M^{a}$, **Lorenzo Hernández, $M^{a}$ Piedad *Soria Suárez, $\mathrm{M}^{\mathrm{a}}$ Isabel}

\author{
*Enfermera. UGC Cirugía General y Especialidades del HGB de Baza Granada. E-mail: \\ rosah_c@hotmail.com ** Enfermera. Servicio de Urgencias y Emergencias de Águilas. Murcia.
}

Palabras clave: Bifosfonatos; Mieloma Múltiple: Ácido Zoledrónico; Metástasis Óseas; Dolor óseo Keywords: Bisphosphonates; Multiple Myeloma; Zoledronic Acid; Bone Methastasis; Bone Pain.

\section{RESUMEN}

El objetivo principal del estudio del caso clínico fue determinar si el uso de los bifosfonatos en el tratamiento del Mieloma Múltiple es efectivo para conseguir disminución del dolor en el paciente y para valorar la disminución de hipercalcemia. Para ello se ha estudiado un caso clínico de una paciente sometida a tratamiento de quimioterapia y coadyuvado con tratamiento con Zometa ${ }^{\circledR}$ en el Hospital Comarcal de Baza (Granada), durante las primeras sesiones del tratamiento, obteniéndose resultados favorables ya que se evidenció disminución en la incidencia de hipercalcemia y reducción del dolor. Tras una exhaustiva valoración del paciente, se seleccionaron 3 diagnósticos de enfermería, y se planificaron las intervenciones más apropiadas para cada uno de ellos, utilizando la taxonomía NANDA, NIC y NOC. Durante el tratamiento de la paciente se observaron variaciones en la temperatura corporal poco significativas y náuseas en las primeras sesiones; así como disminución del dolor que conllevó a un menor uso de analgésicos, normalización de cifras de calcio sérico y un aumento en su calidad de vida. Las conclusiones del estudio determinaron un efecto positivo en la administración del Ácido Zoledrónico, ya que se obtuvieron los resultados previstos y los efectos secundarios fueron mínimos, sin necesidad de interrumpir el tratamiento.

\section{ABSTRACT}

The main objective of the case was whether the use of bisphosphonates in the treatment of multiple myeloma is effective for decreasing pain in patient and to evaluate the reduction of hypercalcemia or not. We have studied a case of a patient undergoing chemotherapy and contributed to â Zometa ${ }^{\circledR}$ in the Regional Hospital of Baza (Granada-Spain), during the first session of treatment the patient revealed favourable results as evidenced decreased incidence of hypercalcemia and pain reduction. After an exhaustive assessment of the patient, three nursing diagnoses were selected; the most appropriate interventions for each of these diagnoses were planned, using NANDA taxonomy, NIC and NOC taxonomy.

During treatment the patient had some variation in body temperature and insignificant nausea in the first 
sessions, as well as decreased pain which led to use less analgesics, normalization of serum calcium levels and an increase in her quality of life. The findings of the study identified a positive effect on the administration of Zoledronic Acid as the expected results were obtained and the side effects were minimal, without interrupting the treatment.

\section{INTRODUCCIÓN}

El Mieloma Múltiple (MM) es una enfermedad caracterizada por una proliferación y acúmulo de células neoplásicas plasmáticas en la médula ósea y la producción de la inmunoglobulina monoclonal, afectando a múltiples localizaciones. La proliferación de células plasmáticas da lugar a una reducción del número normal de células sanguíneas, lo que puede conllevar a una destrucción del hueso y daños orgánicos, principalmente en el riñón.

EI MM es el segundo cáncer más frecuente de la sangre, después de los linfomas, representando el $10 \%$ de las neoplasias sanguíneas y el $1 \%$ de todos los cánceres. La incidencia es de aproximadamente 4-6 casos por 100.000 habitantes y año, con mayor incidencia en la población negra, y la edad promedio al diagnóstico es de 65 años (solo $3 \%$ de los casos son menores de 40 años). En los últimos años hay un aumento de los casos, lo cual parece relacionado con el diagnostico más precoz, al realizarse estudios de proteínas en chequeos médicos. En el año 2001 se estima que en España se produjeron 1.716 casos nuevos de MM, 860 en hombres, 856 en mujeres. En total se produjeron 1.554 defunciones, 766 en hombres y 788 en mujeres $^{(1)}$.

El diagnóstico principal del MM se basa en la demostración de invasión medular por elementos plasmáticos malignos, cuyo crecimiento progresivo se acompaña de alza concomitante de la paraproteína secretada y generalmente osteolisis.

El tratamiento se basa en medidas terapéuticas de soporte y tratamiento antitumoral.

1. Medidas de soporte: indicado para aliviar los síntomas y prevenir complicaciones. Consiste en hidratación, prevención de complicaciones óseas, prevención de infecciones, tratamiento del dolor, tratamiento de la anemia y apoyo psicológico. Destacan los bifosfonatos, muy eficaces para prevenir y tratar las complicaciones óseas (fracturas, hipercalcemia...).

2. Tratamiento antitumoral: (quimioterapia, esteroides, radioterapia) para erradicar las células plasmáticas malignas evitando su proliferación. El tratamiento del MM básicamente es quimioterapéutico, quedando la radioterapia como tratamiento paliativo. Entre los efectos secundarios provocados por el tratamiento destacan un aumento de la susceptibilidad a infecciones provocadas por bacterias, afectaciones renales, dolores óseos, anemia, sintomatología neurológica, entre otras.

La supervivencia relativa ajustada por edad a los 5 años del diagnóstico para este tipo de cáncer en nuestro país es de un $41 \%$ en varones y de un $31 \%$ en mujeres. El pronóstico es mejor si la edad al diagnóstico es menor de 45 años, donde se consigue una supervivencia relativa a los 5 años del $65 \%{ }^{(2}$. 
El dolor óseo es el tipo de dolor oncológico más frecuente y constituye un síntoma que afecta considerablemente a la calidad de vida del paciente ${ }^{(3)}$, que generalmente se localiza en costillas y zona lumbar. Es una experiencia sensorial y emocional desagradable, relacionada con daño tisular actual o potencial. Conforme avanza el cáncer, se debilita el sistema óseo, por lo que aumenta el riesgo de fracturas. Cuando los huesos liberan calcio al torrente sanguíneo en cantidades excesivas se produce hipercalcemia que puede ocurrir con metástasis en los huesos debido a la pérdida excesiva ósea (para detectar las lesiones a nivel óseo se usan radiografías, PET y RMN).

La hipercalcemia es la causa más frecuente de insuficiencia renal, pero también contribuye al depósito de sustancia amiloide en los glomérulos, la hiperuricemia, las infecciones repetidas, etc. La hipercalcemia se define como la existencia de cifras elevadas de calcio en sangre, superiores a los valores de referencia, casi siempre > $10,5 \mathrm{mg} / \mathrm{dl}$. Se considera hipercalcemia de origen tumoral cuando la concentración de calcio corregido respecto a la albúmina es $>12 \mathrm{mg} / \mathrm{dl}$.

\section{BIFOSFONATOS: ÁCIDO ZOLEDRÓNICO}

Los bifosfonatos son fármacos utilizados en el tratamiento y prevención de la osteoporosis posmenopáusica y la inducida por corticoides, en la enfermedad de paget y en la hipercalcemia asociada a neoplasias; así mismo están indicados en el tratamiento de las lesiones osteolíticas y del dolor provocado por las mismas (4), siendo usados en hematología en los pacientes con mieloma. Los bifosfonatos son potentes inhibidores de la resorción ósea, con un importante impacto en el tratamiento de las metástasis óseas, puesto que inhiben el reclutamiento y la maduración de los osteoclastos, previenen la migración de monocitos hacia los osteoclastos e interrumpen su ataque a la membrana ósea.

La indicación del tratamiento con bifosfonatos intravenosos suele ser calcemia > 12 $\mathrm{mg} / \mathrm{dl}$ y/o presencia de síntomas.

\section{Clasificación de bifosfonatos}

Los bifosfonatos se pueden clasificar, en función a su mecanismo de acción, en bifosfonatos Nitrogenados o No Nitrogenados, perteneciendo al grupo de Primera o Segunda y Tercera Generación ${ }^{(5)}$.

1. Primera Generación. No contienen nitrógeno (etidronato, clodronato y tiludronato). Se unen a moléculas de ATP que, incorporadas en osteoclastos, llegan a ser citotóxicas para estas células, alterando su función celular y produciendo su apoptosis.

2. Segunda y Tercera Generación. Nitrogenados (pamidronato, alendronato, ibandronato, risedronato y zolendronato). Considerados más potentes que los No Nitrogenados. Inhiben a la farnesil pirofosfatasa sintasa y otros pasos finales de la vía intracelular del mevalonato, cuyo producto final es el colesterol.

El Ácido Zolendrónico es el segundo bifosfonato aprobado para el tratamiento de la osteoporosis vía intravenosa. Aproximadamente el $40 \%$ de una dosis de ácido zolendrónico vía intravenosa se elimina de manera inalterada en orina, y el $60 \%$ de la dosis inyectada se une al tejido óseo, desde donde se libera lentamente a la 
circulación sistémica. Los bifosfonatos permanecen en el hueso hasta que se produce el modelado óseo, en ocasiones durante años; y esto trae como consecuencia que se pueden acumular durante tratamientos reiterados. La vida plasmática media es de 7 días aproximadamente (aunque a veces se ha detectado en plasma hasta 28 días después de administración intravenosa).

\section{Efectos adversos bifosfonatos}

Los bifosfonatos pueden producir efectos adversos a nivel renal, relacionados con la estructura principal que comparten estos fármacos y no con las diferencias en las cadenas laterales. En caso de administración intravenosa estos efectos parecen estar relacionados con la dosis administrada y el tiempo de perfusión, aumentando al aumentar la velocidad de perfusión ${ }^{(6)}$. La administración de altas dosis de los bifosfonatos de primera generación y de menor potencia necesitaba infusiones de larga duración para asegurar la seguridad renal. Sin embargo, los nuevos bifosfonatos nitrogenados de mayor potencia son efectivos a concentraciones mucho más bajas, permitiendo una perfusión intravenosa más corta ${ }^{(7)}$.

En cuanto a otros efectos secundarios con más frecuencia fueron síndrome seudogripal (fiebre, escalofríos, artralgias, mialgias, dolor óseo), fatiga, reacciones gastrointestinales, anemia, debilidad, tos, disnea y edema. Pudiendo aparecer también elevación en los niveles de calcio, magnesio o fósforo en sangre.

Se han comunicado casos de necrosis óseas maxilares y mandibulares, que se presentan tras exodoncias, cirugía endodóntica, e incluso espontáneamente, en pacientes tratados con bifosfonatos ${ }^{(8)}$. En pacientes que presenten factores de riesgo concurrentes, se practicará exploración dental y tratamiento odontológico preventivo apropiado antes del tratamiento con bifosfonatos. Mientras dure el tratamiento, estos pacientes evitarán, en la medida de lo posible, las intervenciones dentales invasivas.

\section{METODOLOGÍA}

El estudio se basa en un caso clínico, donde la muestra fue una mujer de 68 años de edad diagnosticada de Mieloma Múltiple, en estadio avanzado con metástasis óseas. El instrumento utilizado para la recolección de datos fue una historia de salud, estructurada por el examen físico y pruebas complementarias, los patrones funcionales y la información obtenida por la paciente, aplicando así el proceso de atención de enfermería de acuerdo a los diagnósticos encontrados.

\section{RESULTADOS}

Presentamos caso clínico de una mujer de 68 años de edad diagnosticada de Mieloma Múltiple y tratada por el Servicio de Hematología y Hemoterapia del Hospital General Básico de Baza. Presentaba metástasis óseas.

El tratamiento utilizado para el mieloma fue quimioterapia seguido de las sesiones de ácido zoledrónico (Zometa®) aplicadas cada 28 días. La aplicación de Zometa® (4mg) se inició en Mayo de 2010 y se siguió la evolución de la paciente durante las primeras 6 semanas.

El medicamento se administraba diluido en 100 cc de Suero Fisiológico, siendo el tiempo en infusión de 15-30 minutos. Previa infusión de la medicación intravenosa, se 
le daba a la paciente un comprimido de calcio vía oral (por indicación médica también se complementaba el tratamiento con suplementos de vitamina D cada 24 horas).

Antes de iniciar el tratamiento con ácido zoledrónico, se le realizó estudio bioquímico para posterior comparación de datos (Tabla 1).

Tabla 1. Analítica antes del tratamiento con ácido zoledrónico

\begin{tabular}{|c|c|}
\hline Sodio & $134 \mathrm{meq} / \mathrm{l}$ \\
\hline Creatinina & $2 \mathrm{mg} / \mathrm{dl}$ \\
\hline Proteinuria & + \\
\hline Calcio sérico & $14,3 \mathrm{mg} / \mathrm{dl}$ \\
\hline
\end{tabular}

El control de la creatinina era fundamental una vez iniciado el tratamiento, para monitorizar la función renal ante posibles complicaciones, que hubieran supuesto la interrupción del tratamiento. Esto no sucedió durante las 6 semanas iniciales del tratamiento.

\section{DIAGNÓSTICOS NANDA}

Tras la valoración de la paciente se seleccionaron 3 diagnósticos de enfermería, y se planificaron las intervenciones enfermeras más apropiadas para cada uno de ellos, utilizando la taxonomía NANDA ${ }^{(9)}$, NIC ${ }^{(10)}$ y NOC ${ }^{(11)}$.

1 - Dolor crónico relacionado con las metástasis tumorales (00133)

Resultados (NOC): Control del dolor (1605)

Intervenciones (NIC): 1- Manejo del dolor (1400)

\section{Actuaciones:}

1-Manejo del dolor (1400)

- Se le proporcionó a la paciente educación sanitaria sobre factores que intervienen directamente en el control del dolor.

-Se le administraron analgésicos en el momento de la administración del ácido zoledrónico en las sesiones en las que precisó, en su caso, se administró paracetamol 1 gramo vía intravenosa.

- $\quad$-Se le pautaron analgésicos para la administración en su domicilio cuando el dolor no cesara y se le enseñaron criterios para que ella misma valorara el grado de dolor según la Escala Visual Analógica de la Intensidad del dolor (EVA) y la terapia de medicamentos según dicho grado en cada momento. 
2- Riesgo de infección relacionado con procedimientos invasivos (00004)

Resultados (NOC): Conocimiento: control de la infección (1807)

Control del riesgo (1902)

Intervenciones (NIC): 1-Mantenimiento de dispositivos de acceso venoso DAV (2440)

2-Vigilancia (6650)

\section{Actuaciones}

1-Mantenimiento de dispositivos de acceso venoso (DAV) (2440)

-Se informó minuciosamente a la paciente sobre el procedimiento que se iba a realizar, y la técnica se realizó de manera aséptica

-Se le enseño a valorar efectos secundarios a infección local o sistémica (dolor, tumefacción, fiebre malestar).

-Se le instruyó a la paciente y a su marido sobre el cuidado del DAV.

2-Vigilancia (6650)

-Se controlaron las constantes en todas las sesiones de administración del ácido zoledrónico.

-Vigilamos la oxigenación de los tejidos y la perfusión tisular de los mismos.

-Se le recomendó que entre las sesiones realizara controles periódicos de tensión arterial y temperatura y que si tenía alguna alteración de las mismas se pusiera en contacto con el Departamento de Hematología del Hospital.

3- Intolerancia a la actividad relacionada con los efectos adversos del tratamiento (00092)

Resultados (NOC): Tolerancia a la actividad (0005)

Conservación de la energía (0002)

Intervenciones (NIC): 1-Manejo de la energía (0180)

Actuaciones

1-Manejo de la energía (0180)

-Le enseñamos a la paciente a observar la aparición de fatiga física o emocional y le dimos pautas para una correcta expresión verbal de sus emociones (tanto a ella como a su marido)

-Se le facilitó una dieta para control de ingesta de nutrientes y con esto evitar un estado de desnutrición o carencia 
-Le recomendamos alternancia de periodos de actividad con los de descanso y sueño para evitar los periodos de fatiga.

\section{Evolución}

Durante la aplicación del tratamiento se realizó un control de constantes, en las que tensión arterial, frecuencia cardíaca, saturación de oxígeno, y glucemia capilar se encontraban dentro de la normalidad.

De los efectos secundarios del tratamiento solo cabe destacar variación de temperatura (Gráfico 1) que se producía en el momento del tratamiento y en algunos casos, la paciente los describía a las horas de la infusión intravenosa del ácido zolendrónico, es decir, eran efectos leves a corto plazo, no se observaron efectos a largo plazo (como osteonecrosis mandibular). La paciente presentó nauseas durante las dos primeras sesiones, un efecto indeseado que quedó en segundo plano por su escasa duración.

\section{Gráfico 1: Variación de temperatura durante las sesiones}

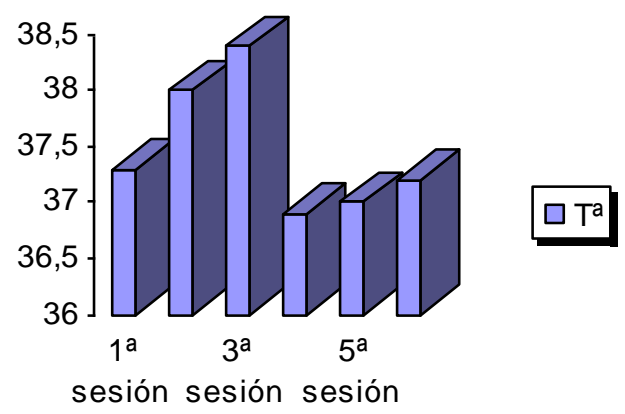

Lo más significativo del tratamiento observado durante estas primeras sesiones, es que la paciente necesitaba menos dosis de medicación analgésica de rescate, con una disminución del dolor debido al tratamiento con ácido zoledrónico (Gráfico 2), lo que iba acompañado de un aumento de su calidad de vida y la normalización de las cifras de calcio sérico desde la primera aplicación del medicamento. 
Gráfico 2: Evolución del nivel del dolor valorado por la paciente (usando la escala EVA)

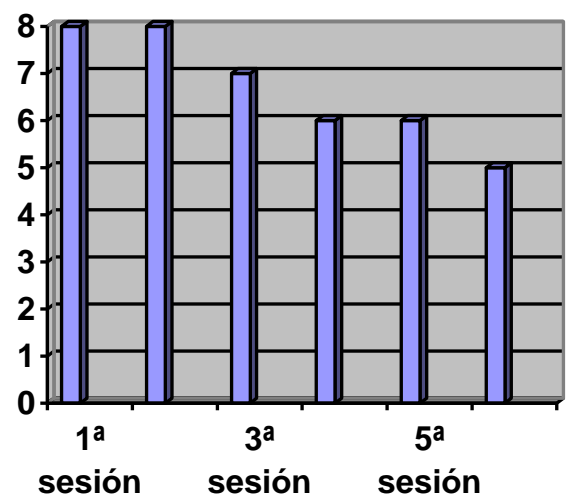

Dolor medido con EVA

Antes del tratamiento, los dolores que padecía eran bastante intensos, referidos sobre todo en espalda, zonas lumbares y más apreciables durante las horas nocturnas.

\section{DISCUSIÓN}

En pacientes diagnosticados por metástasis óseas es importante establecer el inicio del tratamiento y duración con ácido zoledrónico durante períodos superiores a 6 meses o a lo largo de la evolución clínica, en función de la respuesta terapéutica del paciente $^{(3)}$. Este bifosfonato presenta una potencia terapéutica mayor al resto, consiguiendo controlar el dolor óseo, el riesgo de fracturas y frenar el desarrollo de nuevas metástasis óseas con la ayuda de analgésicos, uso de radioterapia, y ciertos isótopos radiactivos ${ }^{(3,12,14,18)}$. Los bifosfonatos han demostrado reducir el dolor óseo mejorando la calidad de vida de los pacientes, disminuyendo, por tanto, el consumo de analgésicos ${ }^{(12,14,17,18)}$. Saban Melina et al., reporta un caso clínico de Enfermedad de Pager ósea, que tras un tiempo de tratamiento con pamidronato no se observa mejoría clínica supliendo el tratamiento por ácido zoledrónico logrando una mejoría clínica y normalización de los niveles de FAL con una sola dosis del fármaco ${ }^{(18)}$.

Cano Castellanos et $\mathrm{al}^{\left({ }^{(13)}\right.}$ han descrito efectos adversos de la función renal con la combinación de bifosfonatos nitrogenados, como el ácido zoledrónico intravenoso y talidomida, al usar una dosis y velocidad de infusión elevada; para evitar estos daños se recomienda el monitoreo de la función renal, analizando la creatinina antes de cada infusión, para la posterior modificación de la dosis, si fuera necesario; no debiendo administrarse ácido zoledrónico si la creatinina es mayor de $265 \mu \mathrm{mol} / \mathrm{L}$..

José María Peña et al. ${ }^{(15)}$ describen caso de una paciente con antecedentes de cáncer de mama, que presentó fracaso renal agudo severo asociado a la utilización de ácido zoledrónico, requiriendo tratamiento con hemodiálisis durante un mes para estabilizar la creatinina sérica. Sin embargo, tras una revisión de ensayos aleatorios realizados por Cochrane ${ }^{(14)}$ refieren que no hubo efectos adversos significativos asociados con la administración de bifosfonatos. Para el manejo seguro del ácido zoledrónico es importante monitorizar la función renal, asegurar una adecuada hidratación y suspender el tratamiento si se detecta deterioro de la función renal ${ }^{(15)}$

Soriano García Jorge $L$ et al,. refieren que a pesar de observar un aumento de la creatinina entre los pacientes tratados con ácido zoledrónico (4 mg) cada 3-4 
semanas durante 2 años, no se observan incrementos severos que modifiquen el grado de toxicidad; siendo estos efectos previsibles y dependientes de la dosis y velocidad de infusión ${ }^{(17)}$.

El ácido zoledrónico ha demostrado ser eficaz en la reducción de la hipercalcemia (nivel de evidencia A), así como es un 16\% más eficaz que el pamidronato para reducir las complicaciones relaciones con el esqueleto. Es útil en el control del dolor óseo asociado a la afectación ósea en pacientes con MM, debiendo acompañarse de tratamiento analgésico de acuerdo a las recomendaciones de la OMS (nivel de evidencia B) ${ }^{(12)}$.

Los efectos adversos producidos por el tratamiento con ácido zoledrónico son variados y poco relevantes, por lo que no suponen contraindicación; entre ellos pueden aparecer náuseas, vómitos y diarrea tras las primeras dosis ${ }^{(12)}$ siendo bien tolerado por los pacientes. Reacciones como cuadros pseudogripales, fiebre, escalofríos, artromialgias y dolores óseos pueden presentarse tras la primera infusión, siendo autolimitados, durante los 3 primeros días de la administración, resolviéndose la mayoría a los 4 días del comienzo del síntoma ${ }^{(16)}$ por lo que no se contraindica la continuación del tratamiento ${ }^{(12,16)}$.

La prevención es la mejor forma de abordar la osteonecrosis maxilar, implicando a todos los profesionales sanitarios, especialmente los odontólogos, hematólogos, cirujanos maxilares deben ser conscientes del riesgo de aparición de este efecto secundario al tratamiento con ácido zoledrónico ${ }^{(4)}$. Autores como Anguita Timoleón et al, (19) reportan caso de osteonecrosis maxilar a los 36 meses de tratamiento con ácido zoledrónico (dosis acumulada de $136 \mathrm{mg}$ ) sugiriendo un aumento de la incidencia con el tiempo de uso y dosis acumulada. Siendo necesario su uso para controlar los problemas asociados al MM no limitando su uso la posibilidad de complicaciones, requiriendo establecer medidas de prevención y tratamiento. En cuanto a la osteonecrosis maxilar, mientras no se compruebe la cicatrización completa de la lesión, se debe suprimir la administración de los bifosfonatos (nivel de evidencia $\mathrm{C}$ ), suspendiendo temporalmente el tratamiento con bifosfonatos 2-3 meses antes de la realización de algún procedimiento invasivo (nivel de videncia D), recomendando sólo su administración únicamente si el balance riesgo-beneficio es claramente favorable (nivel de videncia D) ${ }^{(11)}$. Es de importancia la realización de una monitorización bucodental anualmente (nivel de evidencia D) ${ }^{(12)}$, así como realizar cualquier intervención odontológica previa a iniciar el tratamiento con ácido zoledrónico ${ }^{(17)}$.

\section{CONCLUSIÓN}

El Ácido Zoledrónico, como bifosfonato nitrogenado, es un inhibidor específico de la actividad osteoclástica, siendo de gran importancia en el tratamiento del Mieloma Múltiple y las metástasis óseas derivadas del cáncer, puesto que se consiguen resultados favorables al disminuir el número de metástasis y retrasar su evolución, además de ayudar en el control del dolor óseo, el riesgo de fracturas y consigue normalizar las cifras de calcio en sangre.

Los efectos secundarios provocados con el tratamiento fueron menores respecto al beneficio que se alcanza con el uso de dicha medicación. 
El objetivo principal del proceso enfermero tiene como misión cubrir de forma individualizada las necesidades del paciente, facilitando el diagnóstico y tratamiento de los problemas de salud, teniendo en cuenta al paciente de forma holística. Con la puesta en marcha del plan de cuidados se facilita el trabajo del personal sanitario, teniendo conocimiento de todo el proceso que se le realiza al paciente, proporcionando de esta manera una correcta continuidad en los cuidados. Así mismo, al tratarse de un proceso de valoración continuo existe un correcto seguimiento del paciente, detectando signos de alerta, para evitar complicaciones mayores.

Tanto para el paciente como para el buen funcionamiento del proceso, es fundamental una buena organización interdisciplinar entre oncólogo, radiólogo, equipo de enfermería, servicio de rehabilitación,.etc. Todo ello repercutirá en la calidad de vida del paciente y mejor pronóstico.

\section{BIBLIOGRAFÍA}

(1) Patronato y el Consejo Científico de la Fundación Leucemia y Linfoma (FLL). LIBRO BLANCO DEL MIELOMA MULTIPLE EN ESPAÑA 2004. [acceso 5 Enero 2013]; Pag 1-117. Disponible en http://www.leucemiaylinfoma.com/Control/Noticias/Documentos/Noticias11.pdf

(2) López-Abente G, Pollán M, Aragonés N, Pérez B, Llácer A, Pérez J, Medrano MJ, Boix R, Diez M, González P, Navas A, Almazán J, Jiménez MT, de Pedro J. Tendencias de la mortalidad en España, 1952-1996. Efecto de la edad, de la cohorte de nacimiento y del periodo de muerte. Instituto de Salud Carlos III. Madrid, 2002.

(3) M. A. Vidal, D. Benitez, L. M. Torres. Revisión de las implicaciones clínicas del ácido zoledrónico en el tratamiento del dolor. Rev. Soc. Esp. del Dolor, 2006. [acceso 5 Enero 2013]; 13 (8): 553-559. Disponible en:

http://scielo.isciii.es/pdf/dolor/v13n8/revision2.pdf

(4) Cuevas Ruiz, MV, Martínez Sancho, I; Miriam Fernández Zubiaga, M; Zamora González, M; Álamo González, O. Osteonecrosis del maxilar en paciente con mieloma múltiple tratado con bifosfonatos. 2006. Rev Electron Biomed / Electron J Biomed,2006 [acceso 5 Enero 2013]; 3:14-28. Disponible en http://www. http://biomed.uninet.edu/2006/n3/mvcuevas.html

(5)Torregrosa JV, Ramos AM. Uso de bifosfonatos en la enfermedad renal crónica. Nefrología (Madr.) 2010; [acceso 27 Mayo 2013] 30(3):288-96. Disponible en: http://scielo.isciii.es/scielo.php?script=sci arttext\&pid=S0211-

69952010000300004\&lng=es.

(6) Bounameaux HM, Schifferli J, Montani JP, et al. Renal failure associated with intravenous diphosphonates. Lancet 1983; 1(8322): 471.

(7) Major PP, Coleman RE. Zoledronic acid in the treatment of hypercalcemia of malignancy: results of the international clinical development program. Semin Oncol.2001;28(2 Suppl 6): 17-24.

(8) García F, Torres E, Pereira M, Jiménez R, Torres D, Gutiérrez JL. Osteonecrosis mandibular relacionada con el uso de bifosfonatos. Protocolo de actuación y casos clínicos. Revista SECIB On Line 2008; 1: 16 - 28. Disponible en:

http://www.secibonline.com/web/pdf/vol1 2008 articulo actualizacion2.pdf

(9) NANDA Internacional. Diagnósticos de enfermería 2009-2011: Definiciones y Clasificación. Barcelona: Wilwy-Blackwell.

(10) Bulechek, G M, Butcher H K, McCloskey J. Clasificación de Intervenciones de

Enfermería (NIC). Barcelona: Ed. Elsevier Mosby. 2009 
(11) Moorhead S, Jonson M, Maas ML, Swanson E. 19. Clasificación de Resultados de Enfermería (NOC). Barcelona: Ed. Elsevier Mosby. 2009

(12) García-Sanz R, Alegre A, Capote FJ, Hernández JM, Rosiñol L, Rubia J. Utilización de bisfosfonatos en pacientes con mieloma múltiple: recomendaciones del comité de expertos del Grupo Español de Mieloma del Programa Español de Tratamientos en Hematología. Med Clin (Barc.), 2010 [acceso 28 Mayo 2013]; 134 (6):268-78. Disponible en: http://www.elsevier.es/es/revistas/medicina-clinica2/utilizacion-bisfosfonatos-pacientes-mieloma-multiple-recomendaciones-comite-

13147824-conferencia-consenso-2010

(13)Cano-Castellanos R, Cedillo-de la Cerda JL, Garcés-Ruíz OM, Gómez-Almaguer D, Limón-Flores A, López-Hernández M. Martínez-Baños D. Guías Mexicanas de diagnóstico y recomendaciones terapéuticas para mieloma múltiple. Hematología, 2010 [acceso 27 Mayo 2013]; 11(1):40-62. Disponible en: http://www.medigraphic.com/pdfs/hematologia/re-2010/re101h.pdf

(14) Mascar R, Redzepovic J, Wheatley K, Camara Clark OA, Miladinovic B, Glasmacher A, Kumar A, Djulbegovic B. Bisphosphonates in multiple myeloma: a network meta-analysis. Cochrane Database of Systematic Reviews 2012, Issue 5. Art. No.: CD003188. DOI: 10.1002/14651858.CD003188.pub3. [acceso 28 Mayo 2013 ]Disponible en: http://onlinelibrary.wiley.com/doi/10.1002/14651858.CD003188.pub3/abstract;jsessio nid=37CC02AEC3B02CA33B151849A471E629.d02t02

(15) Peña JM, Vicente C, Pernaute R, y Vives P. Fracaso renal agudo inducido por ácido zoledrónico (Zometa). Nefrología, 2006. [acceso 28 Mayo 2013 ]; 26 (4): 502503. Disponible en: http://revistanefrologia.com/revistas/P1-E253/P1-E253-S122A3937.pdf

(16)Juárez JC, Brumós LG. Ácido Zoledrónico. Tratamiento de la enfermedad de Paget Informe para la Comisión Farmacoterapéutica del Hospital Universitario Vall d'hebron, 2006. [acceso 28 Mayo 2013 ]. Disponible en:

http://gruposdetrabajo.sefh.es/genesis/genesis/Documents/ZOLEDRONICO huvh 03 07.pdf

(17) Soriano García JL, Batista Albuerne N, Lima Pérez M, González González J, Gutiérrez Rojas A, Luaces Álvarez P. Evaluación del uso de ácido zoledrónico en pacientes con cáncer de mama metastásico a hueso. Rev. Cubana med, 2010 [acceso 28 Mayo 2013]; 49(1): 33-64. Disponible en: http://scielo.sld.cu/scielo.php?script=sci arttext\&pid=S0034-

75232010000100004\&lng=es

(18) Sabán M, Fidalgo S, Díaz CA, Lutfi RJ. Efecto de una sola dosis de ácido zoledrónico en un caso de enfermedad de Paget ósea. Medicina, 2010 (B. Aires) [acceso 28 Mayo 2013]; 70(5):445-448. Disponible en:

http://www.scielo.org.ar/scielo.php?script=sci arttext\&pid=S0025-

76802010000500009\&lng=es

(19) Anguita C Timoleón, Agurto P Jaime, Roa E Iván, Laissle C German. Osteonecrosis asociada al uso de bifosfonatos: A propósito de un caso clínico. Rev. méd. Chile , 2006 [acceso 28 Mayo 2013 ]; 134(9): 161-1165. Disponible en:

http://www.scielo.cl/scielo.php?script=sci_arttext\&pid=S0034-

98872006000900011\&lng=es. doi: 10.4067/S0034-98872006000900011

ISSN 1695-6141

๑) COPYRIGHT Servicio de Publicaciones - Universidad de Murcia 